\title{
NON-EQUILIBRIUM SOFT MATTER PHYSICS
}




\section{Founding Advisor:}

Pierre-Gilles de Gennes

(1932-2007)

Nobel Prize in Physics 1991

Collège de France

Paris, France

\author{
Series Editors: \\ David Andelman \\ Tel-Aviv University \\ Tel-Aviv, Israel \\ Günter Reiter \\ Universität Freiburg \\ Freiburg, Germany
}

\author{
Published: \\ Vol. 1 Polymer Thin Films \\ edited by Ophelia K. C. Tsui and Thomas P. Russell \\ Vol. 2 Polymers, Liquids and Colloids in Electric Fields: \\ Interfacial Instabilities, Orientation and Phase Transitions \\ edited by Yoav Tsori and Ullrich Steiner \\ Vol. 3 Understanding Soft Condensed Matter via Modeling and Computation \\ edited by Wenbing $\mathrm{Hu}$ and An-Chang Shi \\ Vol. 4 Non-Equilibrium Soft Matter Physics \\ edited by Shigeyuki Komura and Takao Ohta
}





\section{Published by}

World Scientific Publishing Co. Pte. Ltd.

5 Toh Tuck Link, Singapore 596224

USA office: 27 Warren Street, Suite 401-402, Hackensack, NJ 07601

UK office: 57 Shelton Street, Covent Garden, London WC2H 9HE

\section{British Library Cataloguing-in-Publication Data \\ A catalogue record for this book is available from the British Library.}

\section{Series in Soft Condensed Matter - Vol. 4 NON-EQUILIBRIUM SOFT MATTER PHYSICS}

Copyright (C) 2012 by World Scientific Publishing Co. Pte. Ltd.

All rights reserved. This book, or parts thereof, may not be reproduced in any form or by any means, electronic or mechanical, including photocopying, recording or any information storage and retrieval system now known or to be invented, without written permission from the Publisher.

For photocopying of material in this volume, please pay a copying fee through the Copyright Clearance Center, Inc., 222 Rosewood Drive, Danvers, MA 01923, USA. In this case permission to photocopy is not required from the publisher.

ISBN-13 978-981-4360-62-3

ISBN-10 981-4360-62-7

Printed in Singapore. 


\section{Foreword}

The study of Soft Condensed Matter has stimulated fruitful interactions between physicists, chemists, and engineers, and is now reaching out to biologists. A broad interdisciplinary community involving all these areas of science has emerged over the last 30 years or so, and with it our knowledge of Soft Condensed Matter has grown considerably with the active investigations of polymers, supramolecular assemblies of designed organic molecules, liquid crystals, colloids, lyotropic systems, emulsions, biopolymers, and biomembranes, among others. Taking into account that research in Soft Condensed Matter involves ideas coming physics, chemistry, materials science as well as biology, this series may form a bridge between all these disciplines with the aim to provide a comprehensive and substantial understanding a broad spectrum of phenomena relevant to Soft Condensed Matter.

The present Book Series, initiated by the late Pierre-Gilles de Gennes, comprises independent book volumes that touch on a wide and diverse range of topics of current interest and importance, covering a large number of diverse aspects, both theoretical and experimental, in all areas of Soft Condensed Matter. These volumes will be edited books on advanced topics with contributions by various authors and monographs in a lighter style, written by experts in the corresponding areas. The Book Series mainly addresses graduate students and junior researchers as an introduction to new fields, but it should also be useful to experienced people who want to obtain a general idea on a certain topic or may consider a change of their field of research. This Book Series aims to provide a comprehensive and instructive overview of all Soft Condensed Matter phenomena.

The present volume of this Book Series, edited by Shigeyuki Komura and Takao Ohta, takes as its central aim an exploration of dynamical and nonequilibrium effects of Soft Condensed Matter. Among others, these include rheology of polymers and liquid crystals, dynamical properties of Langmuir monolayers at the air/water interface, hydrodynamics of membranes and 
twisted filaments as well as dynamics of deformable self-propelled particles and migration of biological cells. Complex non-equilibrium phenomena are explored from microscopic to macroscopic scales. The added complexity of soft matter systems manifests itself by intrinsic multi-scale and hierarchical features producing a wealth of challenging dynamical effects. The seven self-contained and clearly written chapters of this volume can serve both as an introduction to students and novice researchers as well as a useful reference to researchers as it covers state of the art topics in the field of non-equilibrium soft matter.

Within the next years, our Series on Soft Condensed Matter will continuously grow and eventually cover the whole spectrum of phenomena in Soft Condensed Matter. We hope that many interested colleagues and scientists will profit from this book series.

David Andelman and Günter Reiter Series Editors 


\section{Preface}

One of the fundamental characteristic features of soft matter is that it exhibits various mesoscopic structures originating from a large number of internal degrees of freedom of each molecule. Due to such intermediate structures, soft matter can easily be brought into non-equilibrium states and cause non-linear responses by imposing external fields such as an electric field, a mechanical stress or a shear flow. As Volume 4 of the series in Soft Condensed Matter, this book focuses on the non-linear and non-equilibrium properties of soft matter.

In the past years, the disciplines of non-equilibrium physics and soft matter physics have developed somewhat independently. When the systematic research of non-equilibrium systems started in the early 1970's, the main subjects were macroscopic non-linear dissipative systems. RayleighBenard convection, Belousov-Zhabotinsky reaction and crystal growth are typical examples. In the 1990's, new experimental techniques to manipulate meso/nanoscopic structures and to obtain real space-time information were developed such as using atomic force microscopy, laser tweezers and three-dimensional tomography. These achievements produced a new era of non-equilibrium physics which deals with out-of-equilibrium situations at a small scale. In fact, soft matter offers a rich variety of experimental systems that exhibit unique out-of-equilibrium phenomena. In turn, they have motivated further development of non-equilibrium statistical mechanics. Such an academic phase in the last decade promoted the momentum toward studying these two research fields in an interactive manner. This is the central concept of non-equilibrium soft matter physics; a new scientific frontier in the twenty-first century.

Studying non-equilibrium soft matter includes several aspects. (i) In dense polymer systems, for instance, due to a huge internal freedom and a high susceptibility, characteristic structures are formed under external fields. The polymer dynamics such as gel formation is strongly influenced by the microscopic structure itself. This coupling effect in different space- 
time scales is one of the key issues in modern polymer science. (ii) Soft matter often exhibits structural transitions by applying external fields such as an electric field or a shear flow. These morphological transitions lead to a drastic change in the physical properties of the material. It is of great interest and importance to elucidate the underlying kinetic pathways of these different non-equilibrium transitions. (iii) Soft matter driven farfrom-equilibrium manifests striking nonlinear properties as a result of its high sensitivity to external fields. In those circumstances, non-equilibrium fluctuations and structural transitions at a mesoscopic level strongly affect macroscopic spatio-temporal structures. In order to explore these nonequilibrium phenomena, new experimental and theoretical tools from the statistical mechanics point of view have recently been developed.

Yet another exciting and important direction of non-equilibrium soft matter physics is to aim at biology. Living matter consists of various soft materials (hence soft matter complex) and can be regarded as one of the ultimate non-equilibrium systems. Generally speaking, biological systems offer a rich variety of fascinating non-equilibrium phenomena that can be fundamentally understood by means of appropriate physical modeling. In order to describe them by using the language of physics, various methods and accumulated knowledge in soft condensed matter physics are indispensable. We believe that the progression of non-equilibrium soft matter physics will provide us with a promising outcome in the future of biophysics.

Although many independent researchers in the world were consciously aware of the necessity of non-equilibrium soft matter physics, it has been strongly pursued in Japan during the last decade as an intensive scientific stream in physics and chemistry (and some biology). Such a trend was supported by the Grant-in-Aid for Scientific Research on Priority Areas "Creation of non-equilibrium soft matter physics: structure and dynamics of mesoscopic systems" (2006-2010) from the Ministry of Education, Culture, Sports, Science and Technology of Japan. This national project was headed by one of the editors of this book (TO), and involved more than 100 Japanese researchers. Almost 1,000 papers have been published in the last five years out of this project. More importantly, some new concepts such as soft matter composites, active soft matter, or driven soft matter began to emerge. We now believe that the importance of this new field is shared not only by Japanese scientists but also by many soft matter scientists around the world. In fact, several international conferences have been held in the recent years on this subject: "International Symposium on Non-Equilibrium Soft Matter 2010" (August 17-20, 2010, Nara), "Mini- 
Symposium on Non-Equilibrium Soft Matter" (July 5, 2011, Berlin), "Soft Matter Far from Equilibrium" (Gordon Research Conference, August 1419, 2011, New London).

As the editors, our aim in this book is to present some of the recent achievements of non-equilibrium soft matter physics in a comprehensive manner. Although the topics discussed are widespread ranging from microscopic molecular dynamics of polymers to macroscopic behavior of structured fluids or migration of cells, their common features are the formation of mesoscopic and hierarchical structures under non-equilibrium situations. The main purpose of this book is to deliver an overview on the exciting and rapidly growing area of research. We required that each independent chapter should be self-contained. It is our great pleasure if students, professors and researchers find this book a useful guide and resource to the field of non-equilibrium soft matter physics.

This book consists of seven chapters. In Chapter 1, Doi describes Onsager's variational principle which gives a unified framework in formulating various dynamics of soft matter. In Chapter 2, the rheodielectric behavior of polymers, liquid crystals, and soft matter composites is discussed by Watanabe et al. In Chapter 3, Orihara presents experimental study of morphology and rheology of immiscible polymer blends under both electric and shear flow fields using a new system combining a rheometer and a confocal scanning laser microscope. In Chapter 4, using a photosensitive twodimensional monolayer prepared from an azobenzene surfactant, Sagués et al. discuss dynamical properties of the forced Langmuir monolayer. In Chapter 5, Komura et al. describe hydrodynamic effects in multicomponent fluid membranes. The presence of the outer bulk solvent is shown to play an essential role for the membrane dynamics. In Chapter 6, Wada et al. study non-equilibrium twist dynamics of rotationally driven semiflexible polymers and filaments in a viscous fluid by means of analytical and numerical approaches. In the final chapter, Sano et al. present numerical and analytical studies on the dynamics of deformable self-propelled particles. Some experiments revealing the correlation of the shape and the stress distribution in a migrated cell are also discussed. It is important to notice, however, that these topics still do not cover all the aspects of non-equilibrium soft matter physics. Those who are further interested in this field may refer such as to the special section of "Non-equilibrium soft matter" published in Journal of Physics: Condensed Matter, Volume 23, 2011. 
We are grateful to the contributors of this book who have taken enormous time and effort in completing chapters. Special thanks are due to Prof. David Andelman and Prof. Günter Reiter (series editors) who initially gave us the opportunity to edit and contribute to this book. We appreciate the anonymous reviewers who had kindly spent their time and given constructive suggestions for improvements of this book. Finally, this book would not have been published without the help of Mr. Alvin Chong from World Scientific Publishing who has been continuously supporting us in our endeavors. 


\section{Contents}

Foreword $\quad$ v

Preface vii

1. Onsager's Variational Principle in Soft Matter Dynamics 1 M. Doi

2. Rheo-Dielectric Behavior of Soft Matters

H. Watanabe, Y. Matsumiya, K. Horio, Y. Masubuchi and T. Uneyama

3. Morphology and Rheology of Immiscible Polymer Blends in Electric and Shear Flow Fields

H. Orihara

4. Dynamical Aspects of Two-Dimensional Soft Matter

F. Sagués, J. Claret and J. Ignés-Mullol

5. Hydrodynamic Effects in Multicomponent Fluid Membranes

S. Komura, S. Ramachandran and M. Imai

6. Actively Twisted Polymers and Filaments in Biology

H. Wada and R. R. Netz 
7. Dynamics of Deformable Self-Propelled Particles:

Relations with Cell Migration

M. Sano, M. Y. Matsuo and T. Ohta 